\title{
The Nanoscale Mechanism for San Carlos Olivine Carbonation
}

\author{
Youngchul Kim, Ryan Nunez, R. W. Carpenter, Andrew V. G. Chizmeshya and Michael J. McKelvy
}

Arizona State University, P O Box 871704, Tempe, AZ, 85287-1704, USA

Carbon dioxide emissions from fossil fueled power plants are of concern due to their potential to induce global climate change[1]. Mineral carbonation, i.e. reaction of the anthropogenic $\mathrm{CO}_{2}$ in a purpose-built processing plant to produce a geologically stable carbonate is a possible method to reduce emissions. Understanding the carbonation reaction mechanism is essential for process control. We are investigating the nanoscale reaction mechanism in two magnesium silicates: San Carlos olivine and serpentine. We showed earlier that carbonation begins by formation of a reaction layer on olivine surfaces, in our case on [001] single crystal surfaces [2]. We are using the standard USDOE Albany Research Center reaction conditions: isothermal reaction experiments for various times up to $8 \mathrm{hrs}$ in $1 \mathrm{M} \mathrm{NaCl}$ and $0.64 \mathrm{M} \mathrm{Na} \mathrm{HCO}_{3}$ aqueous solution at $185 \mathrm{C}$ under $2200 \mathrm{psi}^{\mathrm{CO}_{2}}$ pressure[3], but without rapid stirring, to preserve reaction layer morphology. The overall carbonation reaction is:

$$
\mathrm{Mg}_{2} \mathrm{SiO}_{4}+2 \mathrm{CO}_{2}=2 \mathrm{MgCO}_{3}+\mathrm{SiO}_{2} \text {. }
$$

Several microscopy techniques were required to provide sufficient insight to understand the mechanism: standard and stereographic surface imaging, HREM and STEM imaging, EELS and EDS nanospectroscopy, and FFT optical diffraction. When the specimens are removed from the reaction vessel it was necessary to clean them, to prevent $\mathrm{NaCl} / \mathrm{NaHCO}_{3}$ crystal formation on their surfaces. The cleaning method affected the surface morphology. After gently rinsing reacted crystals in pure water the surface appeared rumpled, as shown in fig. 1. Stereographic SEM images showed that the light grain-boundary-like features are raised ridges, curling above the featureless surface regions. EDS spectra showed this surface to have a larger $\mathrm{Si} / \mathrm{Mg}$ ratio than olivine. The larger particles are reaction products or debris left from cleaning, not important for this discussion. Specimens that had gone through the same reaction cycle but that were cleaned by sonication in water had the surface morphology shown in fig.2, where the raised ridges are removed. Stereographic images from these surfaces showed that the dark grain-boundary-like features were grooves in the surface; EDS nanospectra showed that the composition at the bottom of the grooves was olivine, but the "islands" between the grooves had higher $\mathrm{Si} / \mathrm{Mg}$ composition ratio than olivine. Cross section specimens were made for HREM, to examine this reaction or passivation layer (PL) on the olivine surface. Frequent cracking of the layer during preparation indicated that significant residual stress was present. The interface between the olivine substrate and the PL is shown in fig.3. The PL is amorphous and its interface with olivine is nearly flat on the nanoscale. NanoEELS showed the PL to have an [O]/[Si] slightly less than 2; the LL peak occurred at $22.8 \pm 0.4 \mathrm{eV}$ and the Si-L edge shape both agree with earlier results for $\mathrm{SiO}_{2}$ [4]. There was a dilute distribution of nanoparticles in the PL which were shown to be $\mathrm{MgCO}_{3}$ by FFT from the HREM images. The average particle size was $\sim 6 \mathrm{~nm}$, and the volume fraction was 0.13 . Fresnel imaging did not reveal porosity in the PL. PL thickness increased with reaction times up to $\sim 400 \AA$. After that, further reaction tends to crack off PL layer islands. That exposes new olivine to the reactant environment.

The reaction mechanism can be understood by considering eqn.(1) and some of the properties of the materials involved. Olivine is an orthosilicate with $\mathrm{O} / \mathrm{Si}=4$, composed of isolated tetrahedra bound together by intervening $\mathrm{Mg}$ cations. When the $\mathrm{Mg}$ leaves to form the carbonate(eqn. 1) the crystal collapses to form amorphous $\mathrm{SiO}_{2}$ PL. Our results show that nearly all the carbonate leaves the PL. We confirmed this using XRD on particulate reaction product left in the reactor. The molar volumes of the solids involved are: $\sim 42,28$, and $26 \mathrm{cc} / \mathrm{mol}$ for olivine, and the carbonate and dioxide, resp. 
Since little carbonate remained in the PL, a large biaxial in-plane tensile stress occurred in the PL, resulting in the observed fractures normal to the interface (fig.2). The under-island bond at the PL/olivine interfaces(fig. 3) causes a large strain gradient in the PL, resulting in bending-momentinduced interface tensile stress (fig. 4) that breaks the islands away to expose new olivine for reaction[5]. The same mechanism controls the serpentine carbonation reaction, we now find. References

1. Novel Approaches to Carbon Management, National Academies Press, Wash.D.C.,2003.

2. Ryan Nunez et al., Microsc Microanal 9(Supp2), 682-3,2003.

3. W.K.O'Connor et al., Proc. $29^{\text {th }}$ Int. Tech. Conf. Coal Util.\& Fuel Sys. (U.S. DOE) 1, 71(2004)

4. W.M. Skiff, et al., Jour. Appl. Phys.64(11), 6238(1988); Jour. Appl. Phys.62(6), 2439(1987).

5. Q-Y. Tong and U. Gösele, Semiconductor Wafer Bonding, J. Wiley \& Sons, New York, 1999.

6.This project was funded by U. S. Department of Energy Grant DE-FG26-01NT41282.

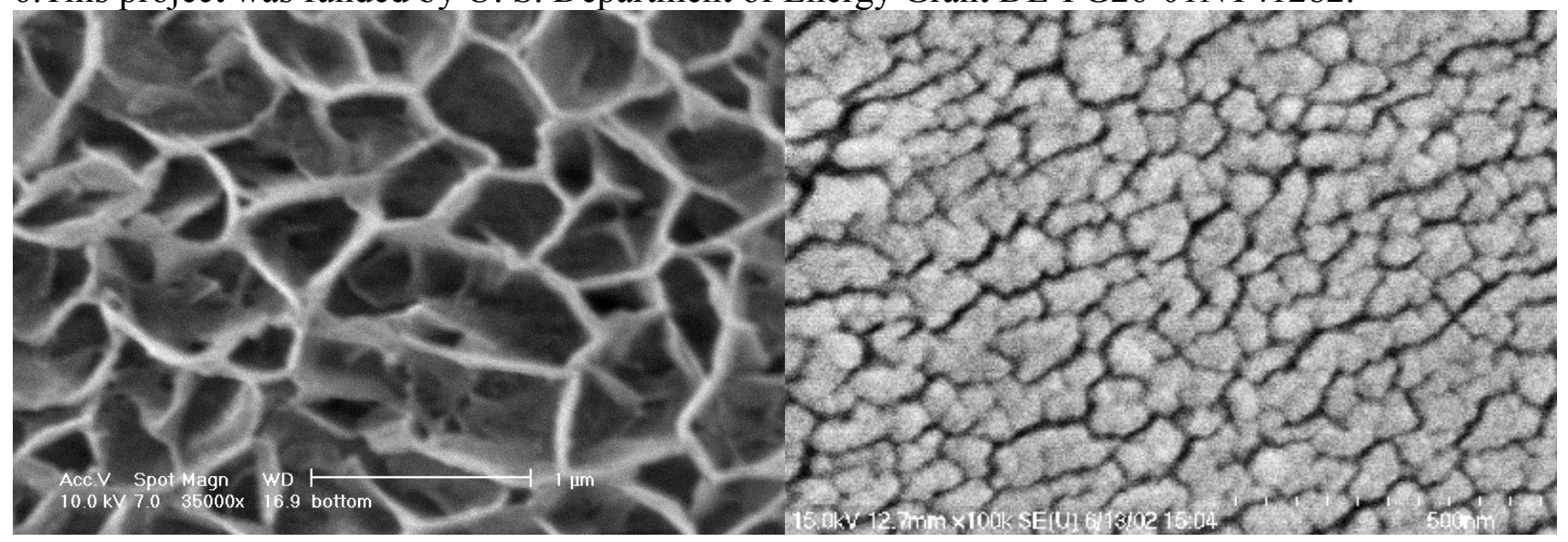

Fig.1.SEM image of olivine reacted for $4 \mathrm{hrs}$. Rinse cleaned only, no sonication. after reaction

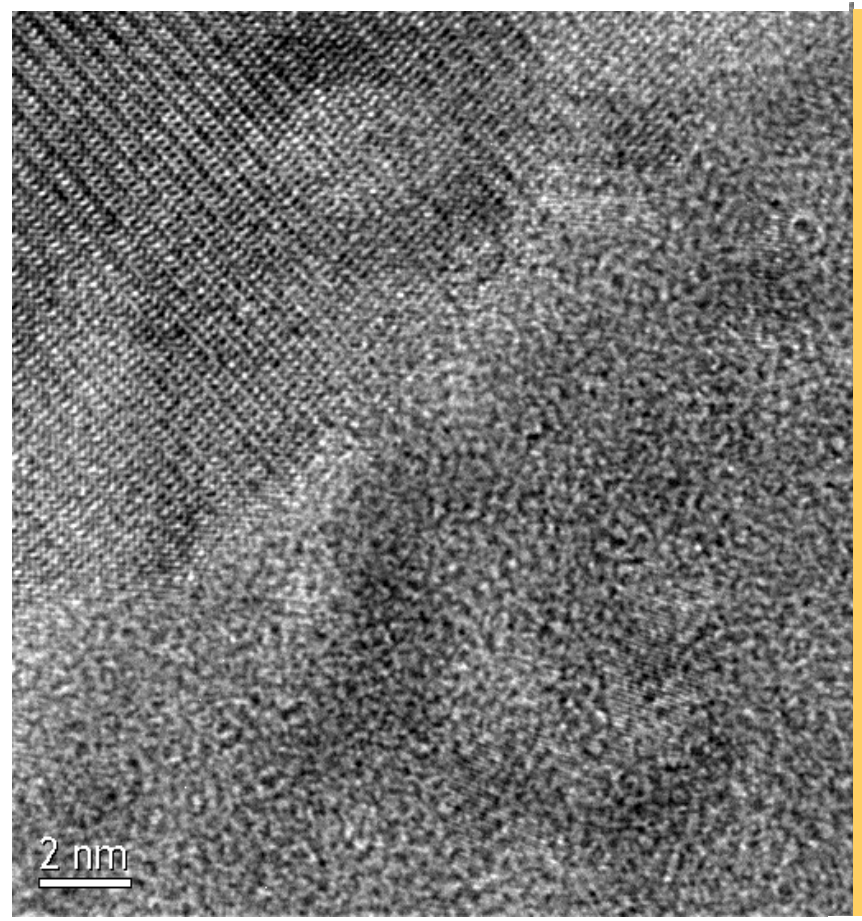

Fig. 3. The olivine/PL interface, under one of the Fig. 4. Reaction model. Brown: Olivine PL islands of fig. 2. 200kV. B $\approx\left[\begin{array}{lll}-1 & 0 & 1\end{array}\right]$ olivine. Wedge polished specimen.
Fig. 2. SEM image of olivine reacted for $4 \mathrm{hrs,}$ and sonication cleaned after reaction.

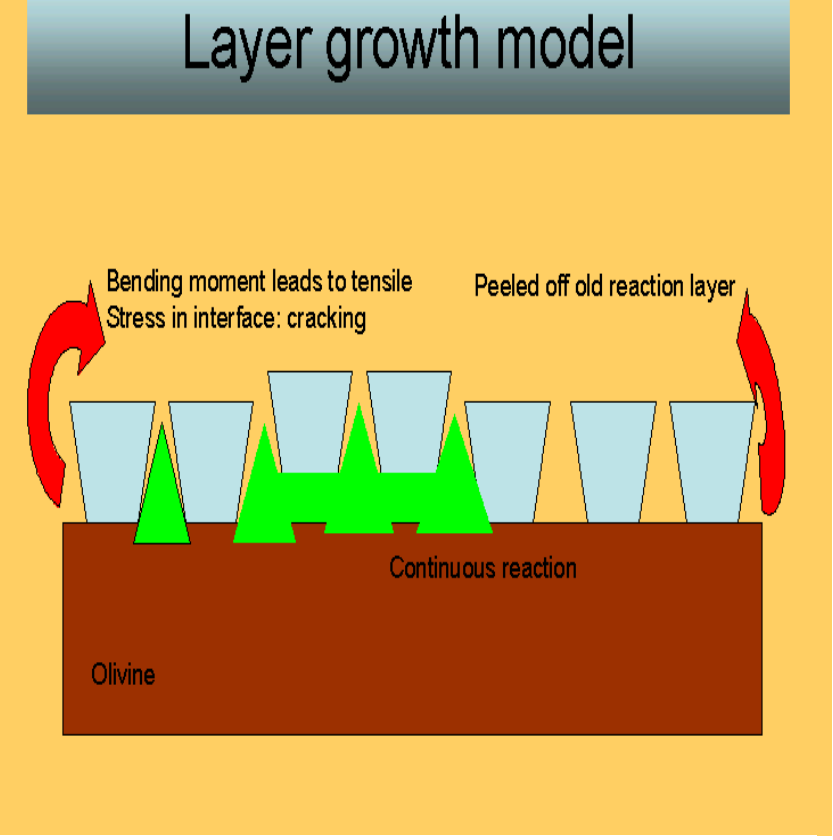

